\title{
Regulation of the expression of phosphoenolpyruvate : carbohydrate phosphotransferase system (PTS) genes in Corynebacterium glutamicum $\mathrm{R}$
}

\author{
Yuya Tanaka, Naoko Okai, Haruhiko Teramoto, Masayuki Inui \\ and Hideaki Yukawa
}

\begin{abstract}
Correspondence
Hideaki Yukawa

mmg-lab@rite.or.jp
\end{abstract}

Received 5 April 2007

Revised 11 September 2007

Accepted 12 September 2007

\author{
Research Institute of Innovative Technology for the Earth (RITE), 9-2, Kizugawadai, Kizugawa, \\ Kyoto 619-0292, Japan
}

\begin{abstract}
The phosphoenolpyruvate: carbohydrate phosphotransferase system (PTS) catalyses the transport of carbohydrates by coupling carbohydrate translocation and phosphorylation. In Corynebacterium glutamicum $\mathrm{R}$, the genes pts $\mathrm{H}$ and pts/ encode general components of the PTS, and genes pts $F$, pts $S$ and pts $G$ each encode fructose-, sucrose- and glucose-specific components of the PTS, respectively. In this study, we examined the mRNA levels of the pts genes in the presence or absence of PTS sugars. Glucose elevated the expression of ptsG, ptsH and $p t s /$ genes, whereas fructose and sucrose induced the expression of all the pts genes examined, i.e. pts $F,-S,-G,-H$ and $-I$. We determined the transcriptional start sites of the pts genes and found that these promoters were activated in the presence of fructose. Disruption of fruR, which is a deoxyribonucleoside repressor (DeoR)-type transcriptional regulator co-transcribed with pts $F$, resulted in enhanced induction of the fructose-pts operon, ptsl, and $p t s \mathrm{H}$ expression in response to fructose, indicating that FruR attenuates the induction of $p t s l, p t s H$ and fructose-pts by fructose.
\end{abstract}

\section{INTRODUCTION}

Corynebacterium glutamicum is a non-pathogenic, highGC, Gram-positive soil bacterium which is widely used for the industrial production of amino acids, notably glutamic acid and lysine (Kinoshita et al., 1957; Ikeda, 2003; Kelle et al., 2005). We are investigating the C. glutamicum $\mathrm{R}$ strain, which can provide high yields of lactate and succinate from sugar (Inui et al., 2004a; Okino et al., 2005). To improve the production of organic acids, understanding the regulatory systems of sugar transport and metabolism is important.

In many bacteria, the phosphoenolpyruvate: carbohydrate phosphotransferase system (PTS) catalyses the transport of carbohydrates by coupling carbohydrate translocation and phosphorylation (Postma et al., 1993; Kotrba et al., 2001a). The PTS consists of two common cytoplasmic proteins, enzyme I and $\mathrm{HPr}$, and an array of sugar-specific enzyme II complexes (EIIs). The phosphoryl group from

Abbreviations: DeoR, deoxyribonucleoside repressor; Ell, enzyme ॥ complex; PTS, phosphoenolpyruvate: carbohydrate phosphotransferase system.

A supplementary figure showing real-time RT-PCR analysis of pts gene expression is available with the online version of this paper. phosphoenolpyruvate (PEP) is sequentially transferred to enzyme I, HPr, EIIs and finally to the substrate as it is translocated across the membrane. In addition to carbohydrate uptake, the PTS regulates the expression of many catabolic genes, depending on its phosphorylation state (Postma et al., 1993; Kotrba et al., 2001a).

Expression of pts genes has been studied in many bacteria (Postma et al., 1993; Vadeboncoeur et al., 2000; Kotrba et al., 2001b; Deutscher et al., 2006). Generally, EII expression is induced in the presence of its substrate sugar. Expression of enzyme I and HPr, encoded by $p t s I$ and $p t s H$, increases in the presence of different PTS sugars. In many bacteria, glucose is reported to be the most effective inducing sugar of ptsI and ptsH expression (De Reuse \& Danchin, 1988; Stülke et al., 1997; Viana et al., 2000; Nothaft et al., 2003). Regulation of pts gene expression is mainly controlled at the stage of transcription initiation or at transcription elongation. The mechanism for control of $p t s$ gene expression differs for the respective pts genes. For example, Mlc protein represses the expression of the ptsHIcrr operon, which encodes general components of PTS, HPr and enzyme I, and the EIIA component of the glucose-PTS in Escherichia coli (Kim et al., 1999; Plumbridge, 1999; Tanaka et al., 1999). This repression is relieved by dephosphorylated glucose-PTS, which is 
generated by the transport of PTS sugar. In Bacillus subtilis, ptsGHI operon expression is controlled by the GlcT antiterminator. GlcT is active in its dephosphorylated form, which is generated by the transport of glucose. Activated GlcT inhibits the rho-independent terminator that precedes the $p t s G \mathrm{ORF}$, and transcription proceeds to the $3^{\prime}$ end of ptsGHI (Stülke et al., 1997).

Expression of the fructose-pts operon is negatively regulated by Cra, which belongs to the GalR-LacI-type regulator family. Repression is relieved by fructose 1-phosphate in $E$. coli. In B. subtilis, expression of the lev operon, which is a fructose-PTS permease forming fructose 6-phosphate, is regulated by the antitermination mechanism mediated by LevR. This operon is also controlled by the CcpA-HPr complex. Ser-phosphorylated HPr binds to CcpA, which belongs to the GalR-LacI-type regulator family, and represses operon transcription. HPr phosphorylation at Ser is catalysed by HPr (Ser) kinase, which is activated in the presence of ATP and fructose 1,6-bisphosphate (Vadeboncoeur et al., 2000). In other low-GC Grampositive bacteria, induction of the fructose-pts operon is mediated by FruR, which is a deoxyribonucleoside repressor (DeoR)-type regulator encoded by the first gene of the fructose-pts operon. In Spiroplasma citri, FruR is an activator, whereas in Streptococcus gordonii and Lactococcus lactis, FruR is a repressor (Gaurivaud et al., 2001; Loo et al., 2003; Barrière et al., 2005). It is proposed that fructose 1-phosphate modulates FruR activity in L. lactis.

In C. glutamicum, the presence of general components (enzyme I and HPr), as well as glucose-, fructose- and sucrose-specific EIIs of the PTS, has been demonstrated in several biochemical and genetic studies (Mori \& Shiio, 1987; Dominguez \& Lindley, 1996; Parche et al., 2001a; Moon et al., 2005), and there are additional pts genes for unknown substrates (NCgl2933 and NCgl2934 of $C$. glutamicum ATCC 13032). C. glutamicum $\mathrm{R}$ has a $\beta$ glucoside-specific PTS in addition to these pts genes (Kotrba et al., 2001b). The genes that encode general components of the PTS are located near the fructosepts gene, which is peculiar to Corynebacterium species (Fig. 3a).

PTS transport assays have revealed that the activity of the C. glutamicum fructose-PTS is induced when cells are cultured in the presence of fructose. In contrast, the activity of glucose-PTS is relatively constant (Mori \& Shiio, 1987; Parche et al., 2001a). Expression of the pts genes of $C$. glutamicum has been examined in only a few studies (Gerstmeir et al., 2003; Kotrba et al., 2003).

In this study, we investigated the expression of the general components of the PTS and the glucose-, fructose- and sucrose-specific EIIs of the PTS. We found that PTS sugars induced the expression of $p t s$ genes. In particular, fructose induced the expression of all the pts genes examined. We also investigated the role of FruR in the control of pts gene expression.

\section{METHODS}

Media and growth conditions. C. glutamicum $\mathrm{R}$ was grown aerobically at $33{ }^{\circ} \mathrm{C}$ in BT minimal medium with Casamino acids (Kotrba et al., 2001b) supplemented with $1.0 \%(\mathrm{w} / \mathrm{v})$ acetate, and various PTS sugars were added at $1.0 \%(\mathrm{w} / \mathrm{v})$ as carbon sources. Antibiotics were added at the following final concentrations: kanamycin, $50 \mu \mathrm{g} \mathrm{ml}^{-1}$; chloramphenicol, $5 \mu \mathrm{g} \mathrm{ml}{ }^{-1}$. Bacterial growth was monitored by determining $\mathrm{OD}_{610}$.

Bacterial strains and plasmids. Construction of recombinant plasmid pCRC800 containing the fruR gene was carried out as follows. The fruR gene was amplified from C. glutamicum R genomic DNA by PCR using primers EcoRI-fruR-20F (5'-GCGGAATTCAACATCAGCGAGGTTAAGCATG- $\left.3^{\prime}\right)$ and HindIII-fruR-840R (5' GCGAAGCTTGCGACAGCGTGGAATCAATAC-3'). The amplified DNA fragment was digested with EcoRI and HindIII, and cloned into the corresponding sites on pCRB1 (Nakata et al., 2003) to construct pCRC800

C. glutamicum R (Yukawa et al., 2007) was used as a wild-type strain. The ptsI-FLAG strain was constructed as follows. First, the $3^{\prime}$ region of the ptsI gene was amplified from C. glutamicum R genomic DNA by PCR using primers SacI-ptsI-707F (5'-CGCGAGCTCGCGGACGAAGCTGAAGCAACCAAG-3') and Salt-1762-25F (5' CGCGTCGACGTATCTGTTGAGCACCAATGAGCTTGAC-3'). The amplified DNA fragment was digested by SacI and SalI, and cloned into the corresponding site on plasmid pCRA925 (Inui et al., 2004b). SpeI and NotI restriction endonuclease sites were introduced at the 3' terminus of the cloned ptsI by PCR using primers SpeI-ptsI-1704R (5'-CGGACTAGTGACTGCTGCGTCGATCACTGC-3') and NotIptsI-1707F (5' -GAGGCGGCCGcACCACTGTTGAGCTAAAAAAGCCTC-3') to construct pCRC801. DNA fragments coding for FLAGepitope tag and chloramphenicol-resistance genes were amplified by PCR using primers SpeI-FLAG-CmF (5'-CGGACTAGTGCAGACTACAAGGATGACGATGACAAGTAAGGCCCTTCCGGTTTTGGGGTAC-3') and Cm-NotIR1 (5'-CGCGCGGCCGCGGCTCTTCCTGTTTTAGAGTGCATTGATC-3'). The amplified DNA fragment was digested by SpeI and NotI, and cloned into the corresponding site on pCRC801 to construct pCRC802. C. glutamicum R was transformed with pCRC802, and $\mathrm{Km}^{\mathrm{S}}$ and $\mathrm{Cm}^{\mathrm{R}}$ colonies were selected. Integration of the FLAG-Cm fragment into the C. glutamicum $\mathrm{R}$ genome by double cross-over was confirmed by colony PCR using primers miniCmL (5'-GCGAAGTGATCTTCCGTTCG-3') and ptsI-1 F (5'-GTGGCTACTGTGGCTGATGT-3'), or mini-CmR (5' -CACGACAGGTTTCCCGACTGG-3') and EcoRI-1761-23F (5'-GCGGAATTCGGCTTTTTGCTTTAAGGAGTGACATG-3').

Disruption of the fruR gene was achieved by transposon-mediated mutagenesis, as described previously (Suzuki et al., 2006). Disruption of the fruR gene was confirmed by DNA sequencing. The transposon was inserted at 463 bases downstream from the $5^{\prime}$ end of the fruR ORF.

The fruR promoter-lacZ strain was constructed as follows. The promoter region of fruR was amplified by PCR using primers SmaIfruR (5'-GCGCCCGGGAAGCAATTGCATGCTGTCTTTCCGTTTG$\left.3^{\prime}\right)$ and SmaI-ptsI (5'-GCGCCCGGGCACATCAGCCACAGTAGCCAC-3'). The amplified fragment was digested with SmaI and cloned into the DraI site of the pCRA741 reporter plasmid, which has been described previously (Inui et al., 2007). These promoter-lac $Z$ fusion plasmids were then digested with $K p n \mathrm{I}$ and SmaI, and the DNA fragment containing the fruR promoter-lac $Z$ gene was cloned into the corresponding site on pHSG398 (Takara) to construct pCRC804. pCRC804 was used to transform wild-type C. glutamicum R or fruRdisruptant cells, and recombinant cells were selected for chloramphenicol resistance. Insertion of the promoter-lac $Z$ fusion gene between CgR0734 and CgR0735 was confirmed by PCR using 
primers LlacZLR-4354F (5'-ATAACCGGGCAGGGGTCTAG-3') and Ind7insert-checkR (5'-GCGTCACGAACAACAGACAGC-3'), or LlacZLR-6425R (5'-CGACGGCCAGTGCCAAGC-3') and Ind7insertcheckF (5'-CGAGACTGGAATTGAGGCTC-3').

Northern blotting analysis. Total RNA was isolated using the RNeasy Kit (Qiagen) from exponentially growing cells $\left(\mathrm{OD}_{610} 1.2\right)$ in BT medium supplemented with $1.0 \%(\mathrm{w} / \mathrm{v})$ carbon source. Ten micrograms of total RNA were resolved by $1.25 \%(w / v)$ agarose gel electrophoresis in the presence of formaldehyde and blotted onto Hybond-N + membranes (GE Healthcare). The mRNAs were visualized using a non-radioactive nucleic acid labelling and detection system (GE Healthcare), according to the procedure specified by the manufacturer. The fluorescein-11-dUTP-labelled DNA probe was synthesized by a random-labelling kit (GE Healthcare). DNA fragments covering each pts gene were used as probes. The signal was scanned by a luminescent image analyser (Fuji model LAS-1000 $\mathrm{CH})$.

Immunoblot analysis. A $10 \mathrm{ml}$ aliquot of cell culture grown to $\mathrm{OD}_{610} 1.0$ was collected by centrifugation, and pellets were mixed with glass beads and $1.0 \mathrm{ml}$ buffer (4\% SDS, $5 \%$ 2-mercaptoethanol, $40 \mathrm{mM}$ Tris/ $\mathrm{HCl}, \mathrm{pH} 6.8,8 \mathrm{M}$ urea, $0.1 \mathrm{mM}$ EDTA). Cells were disrupted by vigorous vortexing and samples were centrifuged. Crude extract $(10 \mu \mathrm{l})$ was loaded onto $0.1 \% \quad(\mathrm{w} / \mathrm{v})$ SDS-10\% (w/v) polyacrylamide gels and electrophoresed. Western blotting was performed using anti-FLAG M2 mAb (Sigma) and horseradish peroxidase-conjugated anti-mouse antibody (GE Healthcare). Chemiluminescence reactions were done using the ECL Plus Western blotting detection system (GE Healthcare). The signal was scanned by a luminescent image analyser (Fuji model LAS-1000 CH).

Primer-extension analysis. Primer extension was carried out using appropriate gene-specific primers (Table 1) and total RNA (10$50 \mu \mathrm{g}$ ). Briefly, $1.5 \mathrm{pmol}$ IRD700-labelled fluorescein primer was hybridized to RNA at $52{ }^{\circ} \mathrm{C}$ for $20 \mathrm{~min}$, and cDNA was synthesized at $42{ }^{\circ} \mathrm{C}$ for $30 \mathrm{~min}$ using the AMV Reverse Transcriptase Primer Extension System (Promega) by the method described by the manufacturer. A $1.65 \mu \mathrm{l}$ aliquot of sample was heat-denatured and loaded onto a denaturing $5.5 \%(\mathrm{w} / \mathrm{v})$ polyacrylamide sequencing gel in parallel with the sequencing ladder and run in the LI-COR DNA sequencer (Aloka model 4000).

Real-time RT-PCR. A total of 20 ng RNA was used as template for analysis of the pts genes, and $0.4 \mathrm{ng}$ was used for analysis of the $16 \mathrm{~S}$ rRNA to generate cDNA and for the subsequent PCR reaction. Each real-time RT-PCR mixture $(20 \mu \mathrm{l})$ contained $500 \mathrm{nM}$ each primer, $12.5 \mu \mathrm{l}$ Power SYBR Green PCR Master Mix, 8 U RNase inhibitor and $5 \mathrm{U}$ MuLV reverse transcriptase (Applied Biosystems). The primers

Table 1. Oligonucleotides used as primers

\begin{tabular}{|ll|}
\hline Primer & \multicolumn{1}{c|}{ Sequence $\left(\mathbf{5}^{\prime} \mathbf{}^{\prime} \mathbf{}^{\prime}\right)$} \\
\hline ptsI-1 & ACATCAGCCACAGTAGCCAC \\
ptsI-2 & AACGGACTCCACCGACAACG \\
ptsH-1 & TTACAGTCTTGGAAGCCATG \\
ptsH-2 & AGCGATGATGGATGCTGGAC \\
ptsG-1 & GTCGTCAGTTTGGACGCCATGTC \\
ptsG-2 & AGTCATCGAAGTAATATTGTCTG \\
fruR-1 & TGTCTTTCCGTTTGGCTGACCATG \\
fruR-2 & GGTGCCAGTAAAGAAGCAATTGC \\
ptsS-1 & CTTCGCCGCCAATGTCGCGCAGGATG \\
ptsS-2 & GCGTAAACGCGTTGCACAGTGTG \\
\hline
\end{tabular}

used in these reactions were: ptsI, ptsIF (5'-GGCTTCGAACATGGAGATGAAC- $3^{\prime}$ ) and ptsIR (5'-TGGCGTAAGGCTGTCATCAAG$\left.3^{\prime}\right)$; and 16S rRNA, 16SF (5' ${ }^{\prime}$ TCGATGCAACGCGAAGAAC-3') and 16SR (5'-GAACCGACCACAAGGGAAAAC-3'). Reactions were performed using the ABI 7500 Fast Real-Time PCR System (Applied Biosystems) with the following cycle parameters: one cycle of $50{ }^{\circ} \mathrm{C}$ for $30 \mathrm{~min}$ and $95{ }^{\circ} \mathrm{C}$ for $10 \mathrm{~min}$, followed by 40 cycles of $95{ }^{\circ} \mathrm{C}$ for $15 \mathrm{~s}$ and $60{ }^{\circ} \mathrm{C}$ for $60 \mathrm{~s}$. The $16 \mathrm{~S}$ rRNA result was used as an internal control.

$\boldsymbol{\beta}$-Galactosidase assay. C. glutamicum $\mathrm{R}$ was grown to $\mathrm{OD}_{610} 1.2$, and $1 \mathrm{ml}$ culture sample was harvested and dissolved in $1 \mathrm{ml} \mathrm{Z} \mathrm{buffer}$ $\left(\mathrm{Na}_{2} \mathrm{HPO}_{4} / \mathrm{NaH}_{2} \mathrm{PO}_{4}\right.$, pH 7.0, $10 \mathrm{mM} \mathrm{KCl}, 1 \mathrm{mM} \mathrm{MgSO}_{4}, 50 \mathrm{mM} \beta$ mercaptoethanol) with $2 \%$ toluene to permeabilize the cells. $\beta$ Galactosidase activity was determined with permeabilized cells by the method described by Miller (1972).

Affinity purification of promoter binding protein. Biotin-labelled ptsI-fruR promoter DNA fragment was generated by PCR using primers promoter-ptsI (5'-GCGCCCGGGCACATCAGCCACAGTAGCCAC- $\left.3^{\prime}\right)$ and bio-promoter-fruR (5'-AAGCAATTGCATGCTGTCTTTCCGTTTG-3'). The $5^{\prime}$ terminus of the bio-promoterfruR primer was labelled with biotin. PCR product (100 pmol) was coupled to $3 \mathrm{mg}$ Dynabeads streptavidin (Dynal). C. glutamicum $\mathrm{R}$ was grown in BT medium supplemented with $2.0 \%$ (w/v) fructose to $\mathrm{OD}_{610} 3.0$, harvested, washed with $50 \mathrm{ml}$ cell wash buffer $(50 \mathrm{mM}$ Tris/ $\mathrm{HCl}, \mathrm{pH} 7.5,50 \mathrm{mM} \mathrm{NaCl}$ ) and suspended in $6 \mathrm{ml}$ binding buffer (50 mM Tris/HCl, pH 7.5, 1 mM EDTA, $10 \%$, w/v, glycerol, $1 \mathrm{mM}$ DTT, $100 \mathrm{mM} \mathrm{NaCl}, 0.05 \%(\mathrm{w} / \mathrm{v})$ Triton X-100) with $1.5 \mathrm{~g}$ glass beads. Cells were disrupted by vigorous vortexing, and cell debris was removed by centrifugation at $12000 \mathrm{~g}$ for $10 \mathrm{~min}$ and then at $50000 \mathrm{~g}$ for $60 \mathrm{~min}$. Supernatants were concentrated by using Amicon Ultra-15 centrifugal filter devices (Millipore) to $1.5 \mathrm{ml}$, and incubated with Dynabeads or $p t s I-f r u R$ promoter-coupled Dynabeads for $2 \mathrm{~h}$ at room temperature. Beads were washed six times with $300 \mu \mathrm{l}$ binding buffer and twice with binding buffer containing $200 \mathrm{mM}$ $\mathrm{NaCl}$. Bound proteins were eluted with $50 \mu \mathrm{l}$ binding buffer containing $1 \mathrm{M} \mathrm{NaCl}$. Eluted fractions of $20 \mu \mathrm{l}$ were loaded onto $0.1 \%(\mathrm{w} / \mathrm{v})$ SDS-12\% (w/v) polyacrylamide gels and electrophoresed. Proteins were transferred to Immobilon-P PVDF membranes (Millipore) and Coomassie Brilliant Blue-stained. The protein bands that appeared only for affinity-purified samples were excised, and the $\mathrm{N}$-terminal protein sequence was determined by APRO Life Science Institute.

\section{RESULTS}

\section{Effects of PTS sugars on pts mRNA expression}

We first analysed the effects of various PTS sugars on pts mRNA levels. Total RNA prepared from C. glutamicum R wild-type cells grown in minimal medium with acetate in the presence or absence of PTS sugars (glucose, fructose and sucrose) was analysed by Northern blotting using probes specific for $p t s F, p t s S, p t s G, p t s H$ and $p t s I$. Acetate was used as a base carbon source, as acetate gives relatively good growth among non-PTS carbon sources in C. glutamicum R, and previous research has resulted in the accumulation of much knowledge of acetate usage in C. glutamicum. We repeated Northern-blotting analysis more than twice and observed the same expression patterns of pts genes.

In the absence of PTS sugars, a very faint band was detected with the $p t s F$ probe (Fig. 1a, lane 1), and addition 
(a)

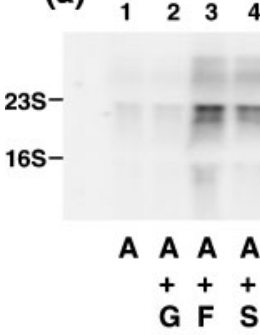

(b)

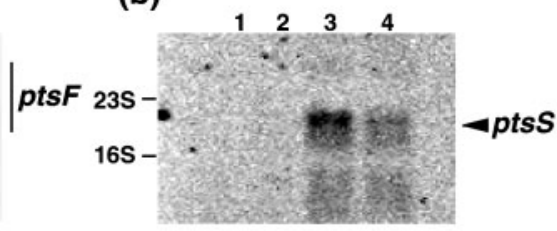

A $\quad$ A A $\mathbf{A}$

$++\dot{t}+$ (c)

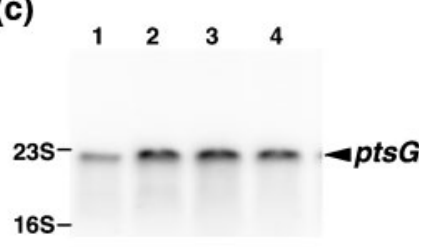

$\begin{array}{rlll}\text { A } & \text { A } & \text { A } & \text { A } \\ & + & + & + \\ & \text { G } & \text { F } & \text { S }\end{array}$

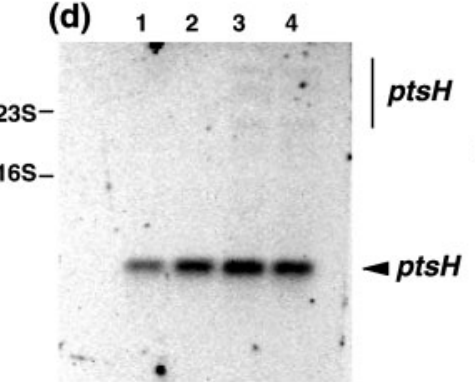

$\begin{array}{cccc}\text { A } & \text { A } & \text { A } \\ + & + & + \\ \text { G } & \mathbf{F} & \mathbf{S}\end{array}$ (e)

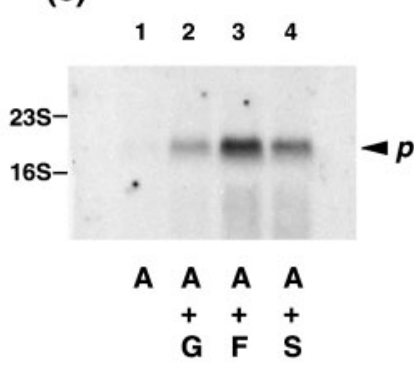

(f)

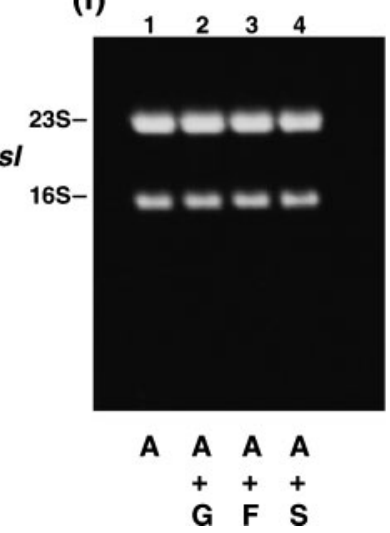

Fig. 1. Effect of various PTS sugars on the expression of pts genes. Total RNA (10 $\mu \mathrm{g})$, prepared from cells grown in BT minimal medium supplemented with Casamino acids and $1.0 \%(\mathrm{w} / \mathrm{v})$ acetate with or without $1.0 \%(\mathrm{w} / \mathrm{v})$ PTS sugars, was subjected to Northern blotting analysis. Abbreviations: A, acetate; G, glucose; F, fructose; S, sucrose. (a) Hybridization with $p t s F$ probe; (b) hybridization with pts $S$ probe; (c) hybridization with ptsG probe; (d) hybridization with ptsH probe; (e) hybridization with pts/ probe. (f) Total RNAs used were stained with ethidium bromide and visualized by UV irradiation. The locations of the 23S rRNA and 16S rRNA are indicated.

of glucose did not increase the level of $p t s F$ mRNA (Fig. 1a, lane 2). In contrast, the addition of fructose increased the expression of ptsF (Fig. 1a, lane 3), and sucrose also increased the $p t s F$ mRNA signal (Fig. la, lane 4). The expression pattern of $p t s S$ was similar to that of $p t s F$, that is, the expression of $p t s S$ was induced by both fructose and sucrose (Fig. 1b). Glucose-specific pts showed a different expression pattern. Significant $p t s G$ mRNA was detected in the absence of a PTS sugar (Fig. 1c, lane 1), and $p t s G$ expression increased in the presence of glucose (Fig. 1c, lane 2). Surprisingly, in the presence of fructose and sucrose, $p t s G$ expression increased to almost the same level as that seen with glucose induction (Fig. 1c, lanes 3 and 4). For general components of the PTS, expression of $p t s H$ was observed in the absence of PTS sugar (Fig. 1d, lane 1), but very little expression was observed for ptsI mRNA (Fig. 1e, lane 1). Both ptsH and ptsI expression increased in the presence of PTS sugar. Fructose was the most effective inducing sugar for $p t s H$ and $p t s I$ expression (Fig. 1d, e, lane 3). The results in Fig. 1(a, d) show that several transcriptional products are present for $p t s F$ and $p t s H$ genes. In summary, fructose and sucrose induced the expression of all $p t s$ genes tested. We observed the induction of $p t s$ genes by PTS sugars when cells were cultured in a rich medium with yeast extract and no added acetate (data not shown), indicating that a decrease in acetate usage is not the signal for the induction of pts gene expression by PTS sugars.

To analyse the effects of PTS sugars on the expression level of PTS proteins, we determined the relative levels of enzyme I by Western blotting. To do this, we introduced a FLAG-tag sequence to the $\mathrm{C}$ terminus of enzyme $\mathrm{I}$, and determined the enzyme I level using an antibody against the FLAG-epitope tag. The enzyme I level increased when external glucose was present (Fig. 2, lanes 4 and 5). A further increase in enzyme I protein level was observed in the presence of fructose (Fig. 2, lane 6). No band corresponding to enzyme I was observed in wild-type cells (Fig. 2, lanes 1-3). This result corresponds to the results of the mRNA study of the ptsI gene (Fig. 1e), and suggests that the regulation of $p t s I$ expression by PTS sugars occurs mainly at the transcriptional level. 


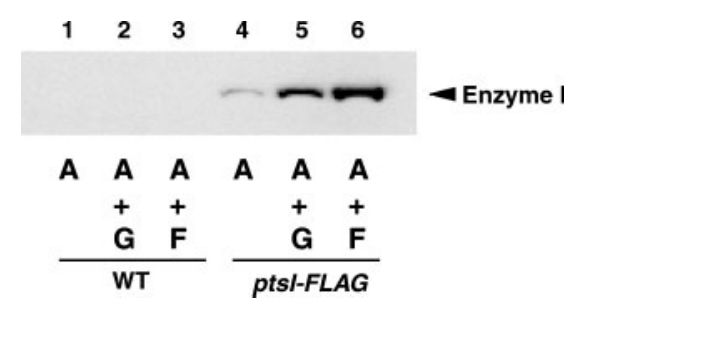

Fig. 2. Immunoblot analysis of cellular enzyme I level. Crude extract equivalent to $O D_{610} 0.01$, prepared from wild-type (WT; lanes 1-4) or pts/-FLAG (lanes 5-8) cells grown in BT minimal medium supplemented with Casamino acids and $1.0 \%(\mathrm{w} / \mathrm{v})$ acetate with or without $1.0 \%(\mathrm{w} / \mathrm{v})$ glucose or $1.0 \%(\mathrm{w} / \mathrm{v})$ fructose, was analysed by Western blotting using anti-FLAG M2 antibody.

\section{Operon structure of the fructose-pts}

According to the genome sequence of $C$. glutamicum $\mathrm{R}$ (Yukawa $e t$ al., 2007), the $p t s F$ and $p t s H$ genes are adjacent to each other (Fig. 3a). The gene order and intergenic spacing suggest that $p t s F$ and $p t s H$ are transcribed as a polycistronic mRNA. $p t s I$ is also located near $p t s F$. Between $p t s F$ and $p t s I$ there is the fruR gene, encoding a protein of 264 aa that shows homology to a member of the DeoR family of transcriptional regulatory proteins, and the $p f k B 1$ gene, encoding a protein of 330 aa that shows homology to fructose-1-phosphate kinase. The fruR, pfkB1, ptsF and $p t s H$ genes are oriented in the same direction, while ptsI and the ORF that encodes a hypothetical protein located downstream of $p t s H$ are oriented in the opposite direction (Fig. 3a). A rho-independent terminator structure is

(a)

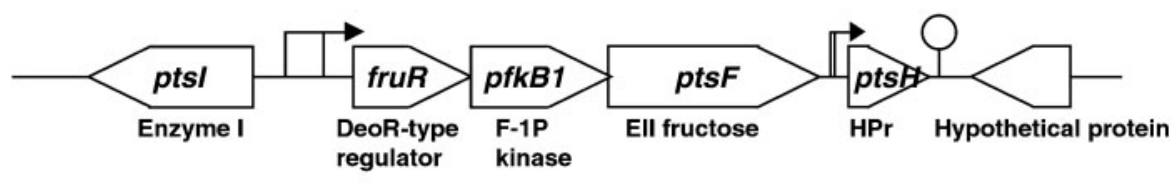

(b)

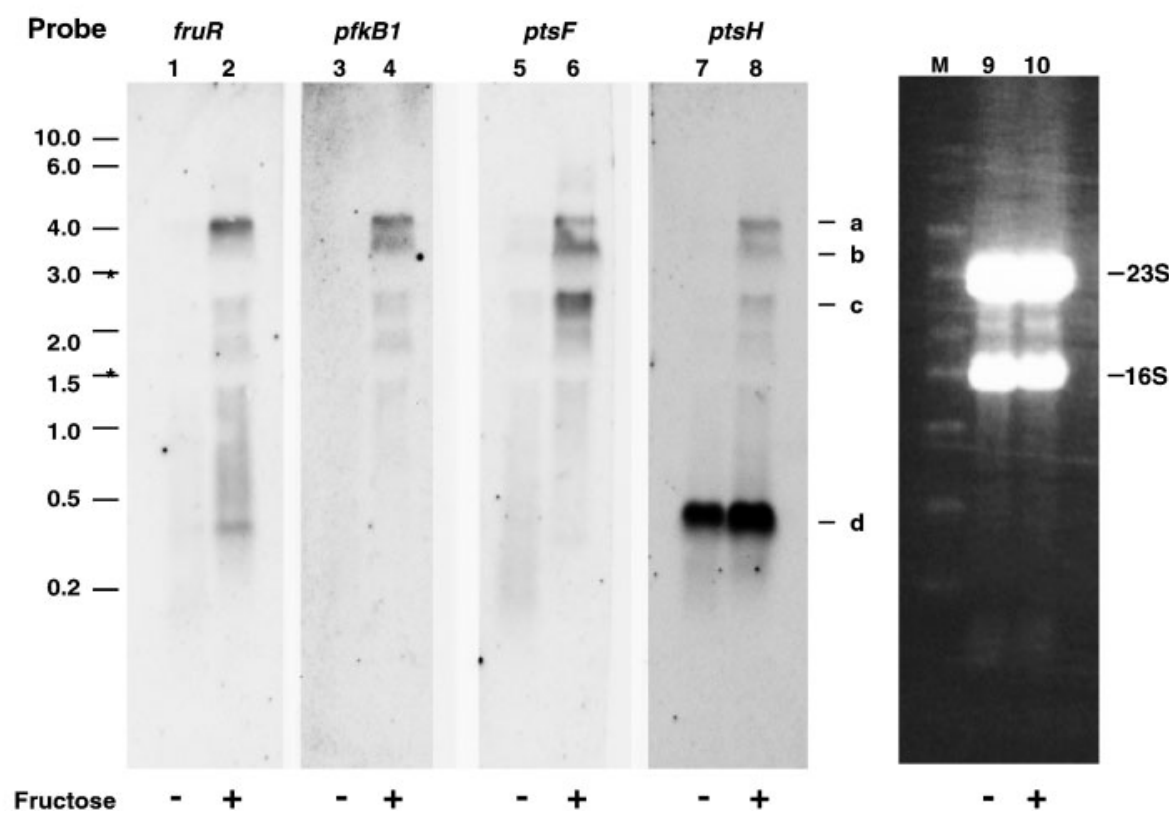

Fig. 3. (a) Genetic organization of the C. glutamicum R fructose-pts operon. Open arrows represent the coding region for the $f r u R, p f k B 1, p t s F$ and $p t s H$ genes. Deduced functions of each gene are indicated below the respective gene. The transcription initiation sites identified in this study (Fig. 4) are indicated by arrows. A putative rho-independent terminator is indicated by a circle. (b) Northern blotting analysis of specific transcripts for fruR, pfkB1, ptsF and ptsH from C. glutamicum R. Total RNA was prepared from cells grown in BT minimal medium supplemented with Casamino acids and $1.0 \%(\mathrm{w} / \mathrm{v})$ acetate, with or without $1.0 \%(w / v)$ fructose. Hybridization was done with probes specific for fruR, pfkB1, pts $F$ and ptsH. Major transcripts are indicated on the right of the panel; RNA standards (Invitrogen) are shown on the left. Asterisks on the left of the Northern blot indicate the position of $23 S$ and $16 S$ rRNAs. The right-hand panel is total RNA stained with ethidium bromide and visualized by UV irradiation. The locations of the $23 \mathrm{~S}$ and $16 \mathrm{~S}$ rRNA are indicated. M, RNA size marker. 
present downstream of the $p t s H$ gene. This gene cluster is also conserved among C. glutamicum ATCC 13032, Corynebacterium diphtheriae and Corynebacterium efficiens (Parche et al., 2001b; Yokota \& Lindley, 2005).

We analysed the operon structure of this region by Northern blotting using probes specific for fruR, $p f k B 1$, $p t s F$ and ptsH (Fig. 3b). A product of about $4.7 \mathrm{~kb}$ (product a) was detected by all four probes, indicating that fruR, $p f k B 1, p t s F$ and $p t s H$ are transcribed as a polycistronic message. Using a $p t s H$ probe, an intense $0.5 \mathrm{~kb}$ transcript (product d) was detected. The $\mathrm{ptsH}$ gene therefore seems to have its own promoter, as the expression pattern of $p t s H$ mRNA was different from that of the other products. It is significant that product $\mathrm{d}$ was seen in the absence of fructose, and the amount of product $\mathrm{d}$ was more abundant than that of other mRNA products. For products b and c, the expression pattern was the same as that for product a, and we observed many bands by primer-extension analysis using a primer specific for $p t s F$. This suggests therefore that these products are the result of the nuclease digestion.

\section{Determination of the transcriptional start sites of the pts genes}

For further detailed analysis of the regulation of pts gene expression, we examined the transcriptional initiation site of each pts gene. Total RNA prepared from wild-type cells grown in a minimal medium in the presence or absence of PTS sugars (glucose or fructose) was analysed by primerextension analysis using fluorescently labelled primers specific for each pts gene (Table 1). The position of the transcriptional start point was confirmed by experiments using two different primers for each pts gene (Table 1). A major transcriptional start site of $p t s H$ ( $p t s H$-P1 promoter) is the $\mathrm{G}$ residue located $102 \mathrm{nt}$ upstream of the start codon of ptsH (Fig. 4a), and we observed a minor signal corresponding to the T residue located $82 \mathrm{nt}$ upstream of the start codon of $p t s H$ ( $p t s H-\mathrm{P} 2$ promoter). Using a $p t s I-$ specific primer, we detected three bands (Fig. $4 \mathrm{~b}, \mathrm{c}$ ). The signals corresponded to $\mathrm{T}$ residues located at 103 and $105 \mathrm{nt}$ upstream of the GTG start codon (ptsI-P1 promoter), and $37 \mathrm{nt}$ upstream of the start codon (ptsI$\mathrm{P} 2$ promoter). Using a $p t s G$-specific primer, we detected a single band corresponding to a $\mathrm{T}$ residue, located $256 \mathrm{nt}$ upstream of the start codon (Fig. 4d). The transcriptional start site of $p t s G$ has recently been reported in $C$. glutamicum ATCC 13032 (Engels \& Wendisch, 2007). Those authors reported the start site at a $\mathrm{G}$ residue located $258 \mathrm{nt}$ upstream of the start codon. The difference from our result may come from the slight difference in DNA sequence at the pst $G$ promoter. The A residue located $262 \mathrm{nt}$ upstream of the start codon in C. glutamicum $\mathrm{R}$ is a $\mathrm{G}$ residue in the ATCC 13032 strain.

To determine the start site of the fructose-specific pts operon, we used a fruR-specific primer. We detected four bands corresponding to $\mathrm{G}$ and $\mathrm{T}$ (fruR-P1 promoter) and
A and T residues (fruR-P2 promoter) located 294, 293, 123 and $126 \mathrm{nt}$, respectively, upstream of the ATG start codon of fruR. A T residue located $62 \mathrm{nt}$ upstream of the start codon was detected using a ptsS primer (Fig. 4g). Nucleotide sequences of the pts promoter regions are compared in Fig. 5. We found putative -10 and -35 hexamers. Comparison of each promoter showed that a TGTTT(TT)G sequence was conserved. The ptsI-P1 promoter region and the fruR-P1 promoter region were found to overlap.

The level of the primer-extension products increased in the presence of fructose (Fig. 4a-g). We also determined the effect of glucose on ptsG mRNA expression, and an increase in $p t s G$ mRNA level was detected (Fig. 4d). These data correspond to the results of Northern blotting assays (Fig. 1).

\section{Effect of fruR gene disruption on the expression of the pts genes}

We were interested in how fructose regulates the expression of pts genes, as fructose has the strongest inducing effect on pts gene expression, and the general component of the PTS is located near the fructose-pts operon, which differs from that of other bacteria. The fruR gene encodes a DeoR-type regulator that is located upstream of $p t s F$ (Fig. 3a). This arrangement is conserved in many bacteria, and the regulator is responsible for the induction of the fructosepts operon (Gaurivaud et al., 2001; Loo et al., 2003; Barriere et al., 2005). Thus, we examined the role of FruR on the expression of the pts genes.

We compared the expression of pts genes in wild-type and fruR cells. In cells grown in the presence of fructose, the mRNA levels of ptsI and $p t s H$ were higher in the fruR mutant than in the wild-type (Fig. 6a, b). In the absence of PTS sugars, or in the presence of glucose, the bands for the mRNAs were faint in both the fruR mutant and the wildtype. The exposure time for detection of bands in the Northern blotting analysis was shorter in Fig. 6 than in Fig. 1 to avoid saturation of the band intensity. These results showed that fructose increased the pts mRNA level in the $f r u R$ strain as in the wild-type, and that the induced level in response to fructose was increased by disruption of fruR. Expression of $p t s G$ was hardly affected by the fruR mutation with all PTS sugars (data not shown).

In the fruR mutant we could not detect the fructose-pts operon mRNA by Northern blotting analysis, probably because of the transposon insertion in the fruR gene. In this regard, we observed that disruption of ptsF completely eliminated the induction of $p t s$ genes in response to fructose. We observed that disruption of $p f k B 1$ completely inhibited growth in the presence of fructose. The phenotypes of the $p t s F$ and $p f k B 1$ mutants were different from those of the fruR mutant. These results suggest that $p t s F$ and $p f k B 1$ are expressed in response to fructose in the fruR cells, although we cannot rule out the possibility that 
(a)

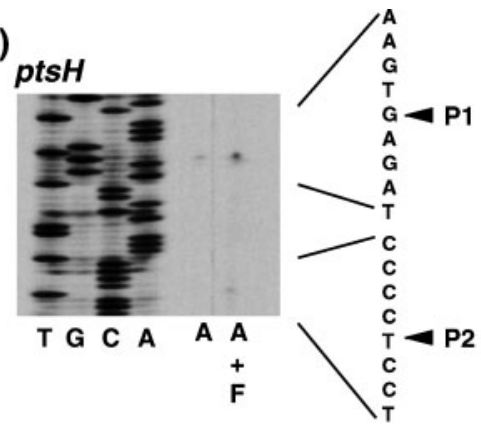

(d)
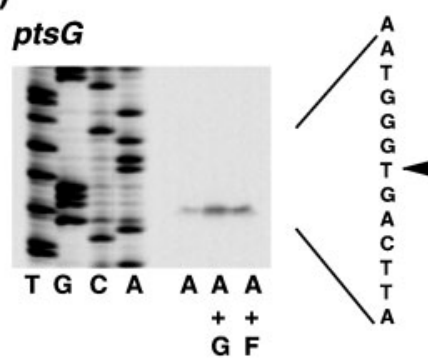

(b)

(e)

(g)

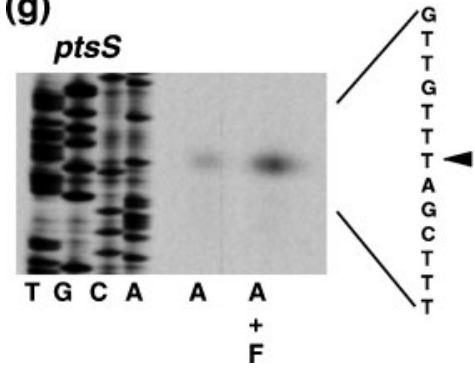

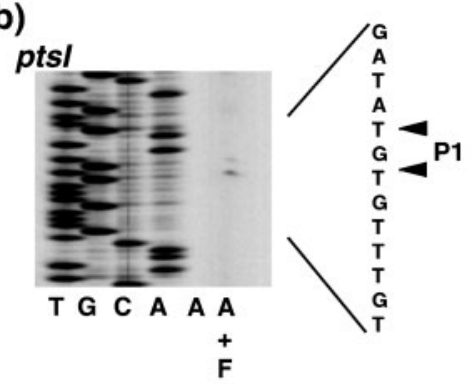

(c)

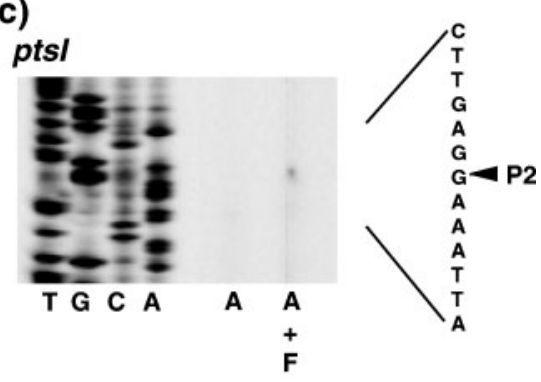

(f)
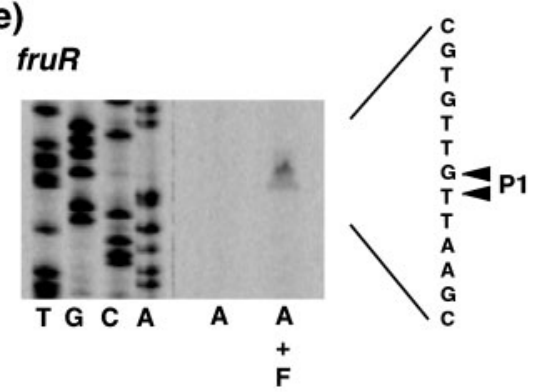
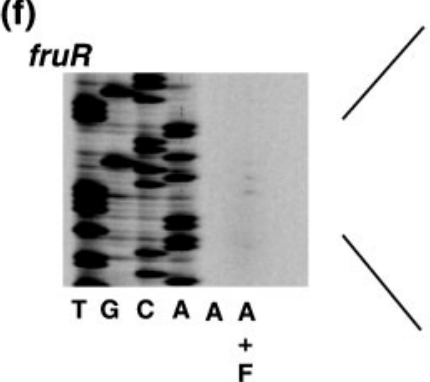

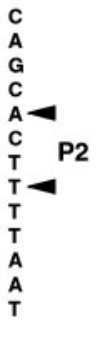

Fig. 4. Determination of the transcriptional initiation sites of the pts genes. Total RNA (10 or $50 \mu \mathrm{g}$ ) isolated from $\mathrm{C}$. glutamicum R cultured in BT medium supplemented with Casamino acids and $1.0 \%(\mathrm{w} / \mathrm{v})$ acetate, with or without $1.0 \%$ (w/v) fructose or glucose, was used for primer-extension analysis with the gene-specific primers listed in Table 1. The transcription initiation sites are indicated by arrows.

the negative effect of disruption of fruR on $p t s F$ and $p f k B 1$ expression affects $p t s$ gene expression indirectly.

We then complemented the fruR mutant with pCRC800, which has the fruR gene expressed under the control of the lac promoter (Fig. $6 \mathrm{~d}$ ). We observed that induction of ptsI by fructose returned to the wild-type level in the strain containing pCRC800 (Fig. 6d, lane 6; Supplementary Fig. S1). These results suggested that FruR functions as a repressor of the pts gene.

To examine whether FruR controls the expression of the fruR-pfkB1-ptsF operon, we constructed a fruR promoterlac $Z$ fusion and then introduced this construct into the wild-type or the fruR mutant in a region of the genome known to be non-essential in C. glutamicum $\mathrm{R}$ (Yukawa et al., 2007). We determined $\beta$-galactosidase activity in the presence or absence of fructose (Fig. 6f). In wild-type cells, a twofold increase in fruR promoter activity was observed in the presence of fructose (Fig. 6f, columns 1 and 2). The induction by fructose seemed weaker than that observed by Northern analysis. This might have been a consequence of the fact that the fruR-lac $Z$ promoter construct included only 135 bp of DNA upstream from the fruR-P1 promoter, which may be insufficient for full promoter activity. Alternatively, the difference might have been caused by post-transcriptional regulation. In fruR-deficient cells, fruR promoter activity increased to a level threefold higher than that in the wild-type (Fig. 6f, columns 3 and 4). In the absence of fructose, fruR promoter activity in fruRdeficient cells was the same as that in the wild-type (Fig. 6f, columns 1 and 3). These results suggested that FruR reduces the induction effect of fructose on the fructose-pts operon. By affinity-purification experiments using ptsI-fruR promoter DNA as a bait (Fig. 6g), we isolated two proteins of about $28 \mathrm{kDa}$ that bound specifically to the promoter region. The determined N- 

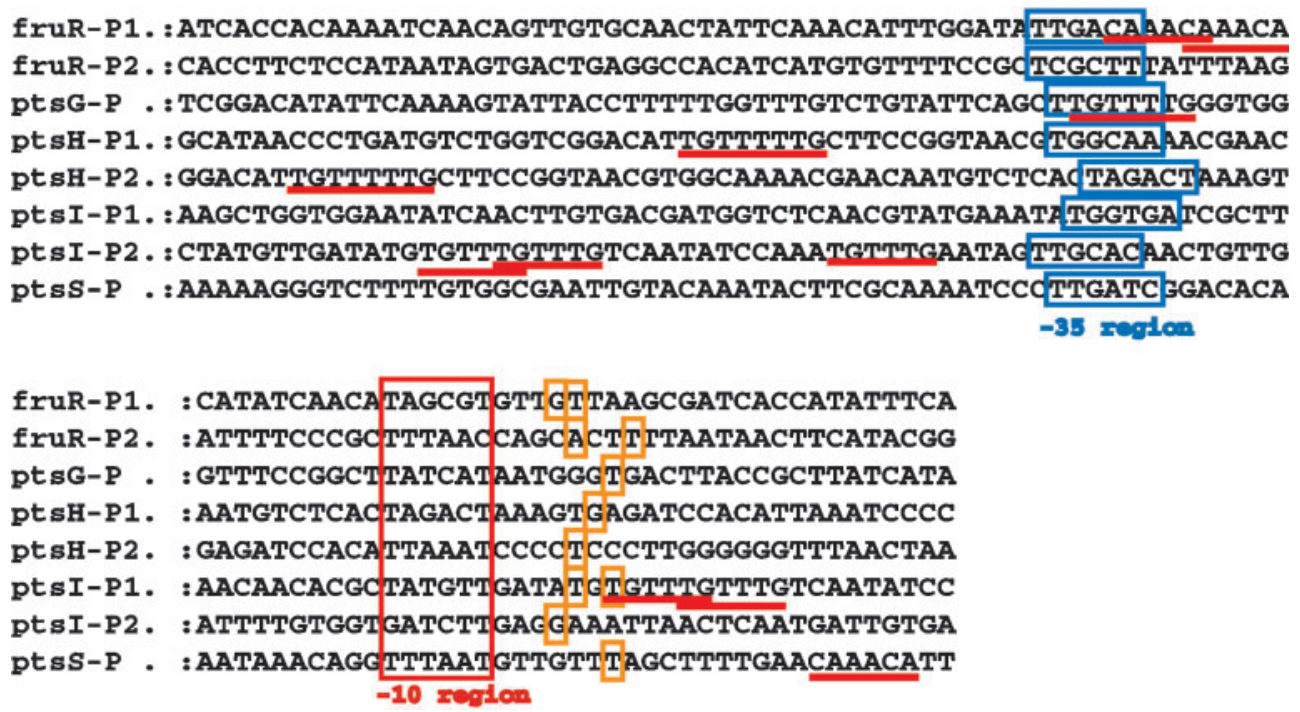

Fig. 5. Alignment of the promoter regions of pts genes. Promoter regions of the fruR, ptsG, ptsH, pts/ and pts $S$ genes are aligned. Transcription start (TS) sites (yellow), -10 regions and -35 regions are boxed. For each promoter, $101 \mathrm{nt}$ are shown; $25 \mathrm{nt}$ downstream of the -10 region and $70 \mathrm{nt}$ upstream of the -10 region. A conserved TGTTT(TT)G sequence or its complement CAAACA sequence is underlined.

terminal amino acid sequence of one protein was VSQTE, which was identical to the $\mathrm{N}$-terminal amino acid sequence of FruR lacking the first amino acid. This result demonstrated that FruR interacts directly with the promoter and suggested that FruR directly represses the expression of the pts gene.

In addition, the second protein we isolated that bound specifically to the promoter region was identified as SugR, which has recently been reported to bind the fruR promoter region (Engels \& Wendisch, 2007).

\section{DISCUSSION}

Sugar transport by the PTS has been demonstrated before in C. glutamicum (Mori \& Shiio, 1987; Dominguez \&
Lindley, 1996; Parche et al., 2001a; Moon et al., 2005). The genes that encode the general PTS and the enzyme II specific for glucose, fructose and sucrose are known. By transport assay, it was demonstrated that glucose-PTS activity is relatively constitutive and that fructose-PTS activity is induced by fructose. However, the regulation of pts gene expression is largely unknown. In this paper we determined the expression patterns of $p t s$ genes in the presence of PTS sugars and determined the location of promoter regions, and finally we investigated the role of FruR on $p t s$ expression.

Expression of $p t s F$ and $p t s S$ showed similar patterns; lowlevel expression of these genes was detected in acetate or glucose cultures, and the expression was induced by both fructose and sucrose (Fig. 1a, b). On the other hand, significant expression of $p t s G$ was observed in the absence

Fig. 6. Effect of fruR mutation on the expression of pts genes. Total RNA (10 $\mu \mathrm{g})$, prepared from wild-type (WT) or a fruR mutant grown in BT minimal medium supplemented with Casamino acids and $1.0 \%(\mathrm{w} / \mathrm{v})$ acetate with or without $1.0 \%(\mathrm{w} / \mathrm{v})$ PTS sugars, was subjected to Northern blotting analysis. Abbreviations: A, acetate; G, glucose; F, fructose. (a) Hybridization with pts/ probe; (b) hybridization with ptsH probe. (c) Total RNAs used in (a) and (b) were stained with ethidium bromide and visualized by UV irradiation; $23 \mathrm{~S}$ and $16 \mathrm{~S}$ rRNA are indicated. (d) Complementation of the fruR mutant. Total RNA was prepared from wild-type cells containing PCRA1 vector or the fruR mutant containing either pCRA1 or pCRC800 plasmid. Northern blotting analysis was performed with the pts/ probe. (e) Total RNAs used in (d) were stained with ethidium bromide and visualized by UV irradiation. (f) Effect of fruR mutation on the activity of the fruR promoter. C. glutamicum $\mathrm{R}$ cells having a fruR promoter-lacZ fusion gene with wild-type fruR or with a fruR gene disruption mutation were grown in BT medium with $1.0 \%(\mathrm{w} / \mathrm{v})$ acetate or $1.0 \%(\mathrm{w} / \mathrm{v})$ fructose. Culture samples were taken at $\mathrm{OD}_{610} 1.2$ and $\beta$-galactosidase activities were determined. Each value is the mean and SD of four independent experiments. ( $\mathrm{g}$ ) Affinity purification of FruR protein bound to the pts/-fruR promoter DNA fragment. Cell extracts of C. glutamicum R grown in BT medium were incubated with magnetic beadsbiotin-labelled pts/-fruR promoter DNA fragment and bound proteins were eluted with $1 \mathrm{M} \mathrm{NaCl}$. In lane 1, proteins were eluted from magnetic beads; in lane 2, proteins were eluted from the pts/-fruR promoter fragment bound to magnetic beads. A molecular mass marker (BioRad) is shown to the left of the panel. 
(a)
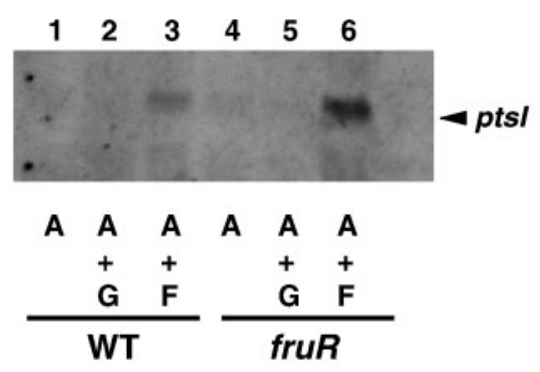

(b)
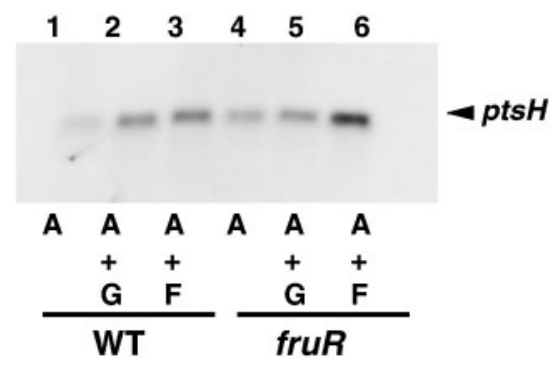

(d)
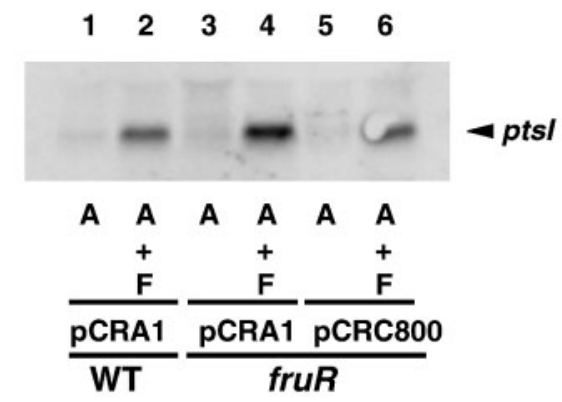

(f)

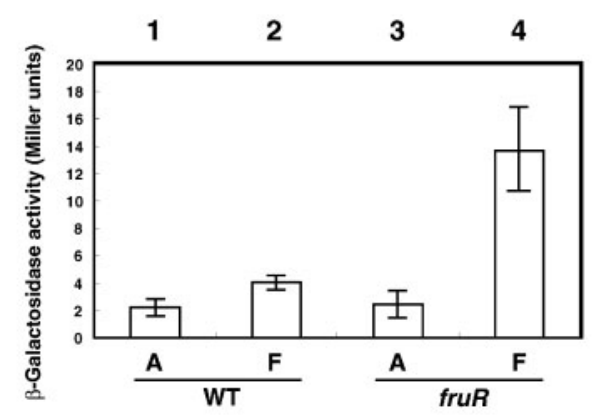

(c)

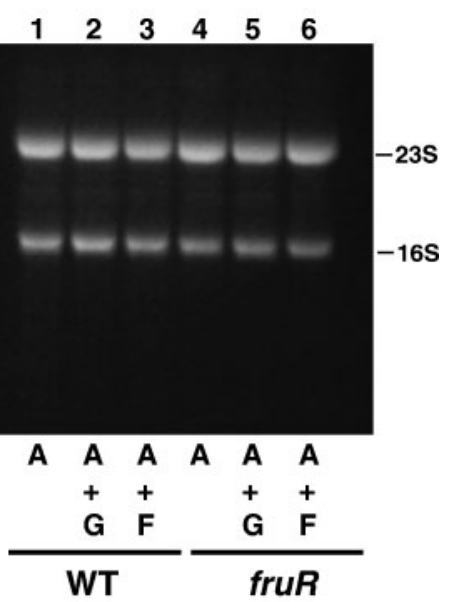

(e)
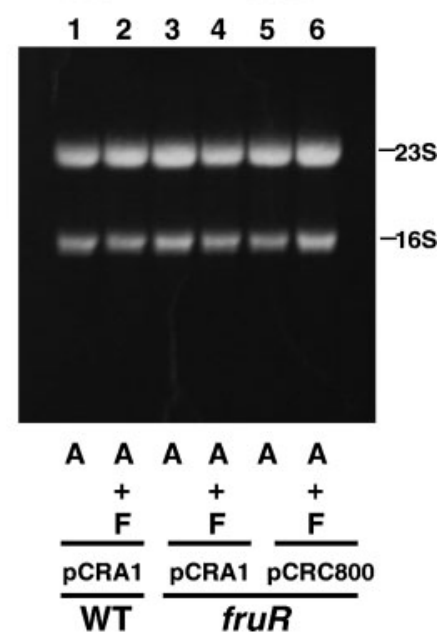

(g)

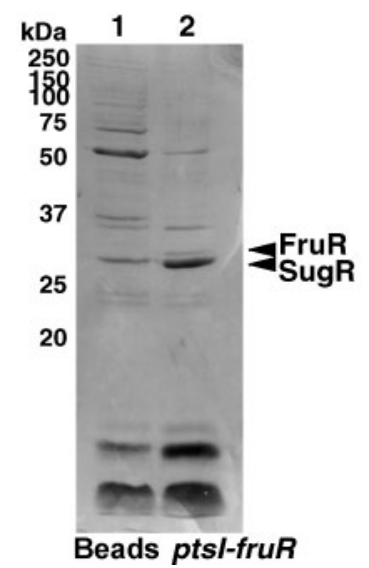

of PTS sugars, and ptsG expression was increased by glucose, fructose and sucrose (Fig. 1c). Glucose-PTS has the ability to transport fructose, although the contribution of glucose-PTS to fructose uptake is only minor (Dominguez et al., 1998; Kiefer et al., 2004). Thus, there might be some advantage for C. glutamicum $\mathrm{R}$ to increase $p t s G$ expression in the presence of fructose. The expression of the general component of the PTS increased in the presence of PTS sugar (Fig. 1d, e). Interestingly, fructose is the most effective inducing sugar, which is different from that which has been observed for other bacteria (De Reuse \& Danchin, 1988; Stülke et al., 1997; Viana et al., 2000; Nothaft et al., 2003). In other bacteria, expression of the general PTS is induced most significantly by glucose. It is also noteworthy that $p t s H$ and $p t s I$ are located near the fructose-pts operon in C. glutamicum, which also differs from the situation in E. coli and B. subtilis, in which ptsH and $p t s I$ are co-transcribed with the glucose-pts genes. In $C$. 
glutamicum, the uptake rate of fructose is faster than that of glucose (Kiefer et al., 2004), which may be explained by the higher expression of the general component of PTS.

In C. glutamicum, sucrose metabolism produces fructose and glucose 6-phosphate, and fructose is exported to the outside of the cell. Fructose re-enters the cell via the fructose-PTS (Dominguez \& Lindley, 1996; Pátek et al., 2003; Moon et al., 2005). This means that the effect of sucrose on the expression of pts genes may be caused by fructose generated inside the cell. By comparing the sucrose induction of pts genes for the wild-type and the ptsF mutant, it might be possible to determine whether sucrose induces $p t s$ gene expression directly or indirectly.

To investigate how pts gene expression is controlled, we determined the transcriptional start site of each pts gene. The nucleotide sequence that resembles the -10 hexamer is located upstream of each transcriptional start site. Alignment of pts promoters through their -10 regions is shown in Fig. 5. The sequence tgngnTA $(\mathrm{c} / \mathrm{t}) \mathrm{aaTgg}$ is proposed as an extended -10 consensus sequence for $C$. glutamicum (Pátek et al., 2003). We compared this sequence with each pts promoter. Core hexamers are well conserved in all pts promoters, especially the first $\mathrm{T}$ and last $\mathrm{T}$, while extended regions are poorly conserved. Only the G residue that is $2 \mathrm{nt}$ upstream of the core hexamer and the $\mathrm{G}$ residue $1 \mathrm{nt}$ downstream from the hexamer are conserved. We also found -35-like sequences that are separated by $15-18 \mathrm{bp}$ from the -10 regions. The hexamer ttGcca is proposed as a -35 consensus sequence (Patek et al., 2003). The first three nucleotides $\mathrm{ttG}$ are well conserved among pts promoters, but the last three nucleotides are less well conserved. Among the pts promoters, the TGTTT(TT)G sequence is conserved, and may be an operator site for the putative common transcriptional regulator for $p t s$. This sequence is unlikely to be the binding site for FruR because FruR does not regulate the expression of $p t s G$, which has the sequence TGTTT(TT)G.

In many bacteria, the gene that encodes the transcriptional regulator located in front of the fructose-pts genes is responsible for the fructose-dependent induction of the fructose-pts operon (Gaurivaud et al., 2001; Loo et al., 2003; Barrière et al., 2005). The regulatory mechanism is either derepression or activation of transcription in the presence of fructose. C. glutamicum also has a regulator FruR for the fructose-pts operon; hence, we determined the effect of fruR disruption on the expression of the pts genes. We found that the expression levels of $p t s I, p t s H$ and the fructose-pts operon increased more in a fruR mutant than in wild-type cells in the presence of fructose, indicating that FruR decreases the induction effect of fructose. This is different from the role of FruR in other bacteria. These results also indicate that transcriptional regulator(s) other than FruR exist, as the increase in pts expression was still observed in the presence of PTS sugar in the fruR mutant. In general, the expression of a catabolic gene is controlled so as not to exceed the metabolic capacity. The role of FruR seems to be to adjust the expression level of pts genes to prevent overflow of PTS sugars.

During the preparation of this manuscript, Engels \& Wendisch (2007) reported that EII components of pts genes ( $p t s G, f r u R-p f k B 1-p t s F$ and $p t s S)$ are regulated by a DeoR-type regulator SugR. SugR represses the expression of EII components of the PTS in the absence of PTS sugar, and addition of PTS sugar relieves this repression. Our results showed that the expression of cytoplasmic components of the PTS ( $p t s H$ and $p t s I$ ) is increased in the presence of PTS sugar. We also found that a conserved motif is found in pts promoters. One possibility is that SugR also controls the expression of $p t s H$ and $p t s I$. We are now investigating whether SugR controls the expression of cytoplasmic components of the PTS.

\section{ACKNOWLEDGEMENTS}

We are grateful to Roy H. Doi (University of California, Davis) for helpful comments on our manuscript. We thank Crispinus A. Omumasaba (RITE) for critical reading of the manuscript. This work was partially supported by a grant from the New Energy and Industrial Technology Development Organization (NEDO).

\section{REFERENCES}

Barrière, C., Veiga-da-Cunha, M., Pons, N., Guédon, E., van Hijum, S. A., Kok, J., Kuipers, O. P., Ehrlich, D. S. \& Renault, P. (2005). Fructose utilization in Lactococcus lactis as a model for low-GC Grampositive bacteria, its regulator, signal, and DNA-binding site. J Bacteriol 187, 3752-3761.

De Reuse, H. \& Danchin, A. (1988). The ptsH, ptsI, and crr genes of the Escherichia coli phosphoenolpyruvate-dependent phosphotransferase system, a complex operon with several modes of transcription. J Bacteriol 170, 3827-3837.

Deutscher, J., Francke, C. \& Postma, P. W. (2006). How phosphotransferase system-related protein phosphorylation regulates carbohydrate metabolism in bacteria. Microbiol Mol Biol Rev 70, 939-1031.

Dominguez, H. \& Lindley, N. D. (1996). Complete sucrose metabolism requires fructose phosphotransferase activity in Corynebacterium glutamicum to ensure phosphorylation of liberated fructose. Appl Environ Microbiol 62, 3878-3880.

Dominguez, H., Rollin, C., Guyonvarch, A., Guerquin-Kern, J.-L., Cocaign-Bousquet, M. \& Lindley, N. D. (1998). Carbon-flux distribution in the central metabolic pathways of Corynebacterium glutamicum during growth on fructose. Eur J Biochem 254, 96-102.

Engels, V. \& Wendisch, V. F. (2007). The DeoR-type regulator SugR represses expression of $p t s G$ in Corynebacterium glutamicum. J Bacteriol 189, 2955-2966.

Gaurivaud, P., Laigret, F., Garnier, M. \& Bove, J. M. (2001). Characterization of FruR as a putative activator of the fructose operon of Spiroplasma citri. FEMS Microbiol Lett 198, 73-78.

Gerstmeir, R., Wendisch, V. F., Schnicke, S., Ruan, H., Farwick, M., Reinscheid, D. \& Eikmanns, B. J. (2003). Acetate metabolism and its regulation in Corynebacterium glutamicum. J Biotechnol 104, 99-122.

Ikeda, M. (2003). Amino acid production processes. Adv Biochem Eng Biotechnol 79, 1-35. 
Inui, M., Murakami, S., Okino, S., Kawaguchi, H., Vertès, A. A. \& Yukawa, H. (2004a). Metabolic analysis of Corynebacterium glutamicum during lactate and succinate productions under oxygen deprivation conditions. J Mol Microbiol Biotechnol 7, 182-196.

Inui, M., Kawaguchi, H., Murakami, S., Vertès, A. A. \& Yukawa, H. (2004b). Metabolic engineering of Corynebacterium glutamicum for fuel ethanol production under oxygen-deprivation conditions $\mathrm{J} \mathrm{Mol}$ Microbiol Biotechnol 8, 243-254.

Inui, M., Suda, M., Okino, S., Nonaka, H., Puskas, G. H., Vertes, A. A. \& Yukawa, H. (2007). Transcriptional profiling of Corynebacterium glutamicum metabolism during organic acid production under oxygen deprivation conditions. Microbiology 153, 2491-2504.

Kelle, R., Hermann, T. \& Bathe, B. (2005). L-Lysine production. In Handbook of Corynebacterium glutamicum, pp. 465-488. Edited by L. Eggeling \& M. Bott. Boca Raton, FL: CRC Press.

Kiefer, P., Heinzle, E., Zelder, O. \& Wittmann, C. (2004). Comparative metabolic flux analysis of lysine-producing Corynebacterium glutamicum cultured on glucose or fructose. Appl Environ Microbiol 70, 229-239.

Kim, S. Y., Nam, T. W., Shin, D., Koo, B. M., Seok, Y. J. \& Ryu, S. (1999). Purification of Mlc and analysis of its effects on the pts expression in Escherichia coli. J Biol Chem 274, 25398-25402.

Kinoshita, S., Udaka, S. \& Shimono, M. (1957). Studies on the amino acid fermentation. I. Production of L-glutamic acid by various microorganisms. J Gen Appl Microbiol 3, 193-205.

Kotrba, P., Inui, M. \& Yukawa, H. (2001a). Bacterial phosphotransferase system (PTS) in carbohydrate uptake and control of carbon metabolism. J Biosci Bioeng 92, 502-517.

Kotrba, P., Inui, M. \& Yukawa, H. (2001b). The ptsI gene encoding enzyme I of the phosphotransferase system of Corynebacterium glutamicum. Biochem Biophys Res Commun 289, 1307-1313.

Kotrba, P., Inui, M. \& Yukawa, H. (2003). A single V317A or V317M substitution in Enzyme II of a newly identified $\beta$-glucoside phosphotransferase and utilization system of Corynebacterium glutamicum R extends its specificity towards cellobiose. Microbiology 149, 1569-1580.

Loo, C. Y., Mitrakul, K. I., Voss, B., Hughes, C. V. \& Ganeshkumar, N. (2003). Involvement of an inducible fructose phosphotransferase operon in Streptococcus gordonii biofilm formation. J Bacteriol 185, 6241-6254.

Miller, J. H. (1972). Experiments in Molecular Genetics. Cold Spring Harbor, NY: Cold Spring Harbor Laboratory.

Moon, M. W., Kim, H. J., Oh, T. K., Shin, C. S., Lee, J. S., Kim, S. J. \& Lee, J. K. (2005). Analyses of enzyme II gene mutants for sugar transport and heterologous expression of fructokinase gene in Corynebacterium glutamicum ATCC 13032. FEMS Microbiol Lett 244, 259-266.

Mori, M. \& Shiio, I. (1987). Phosphoenolpyruvate, sugar phosphotransferase systems and sugar metabolism in Brevibacterium flavum. Agric Biol Chem 51, 2671-2678.

Nakata, K., Inui, M., Kos, P. B., Vertès, A. A. \& Yukawa, H. (2003). Vector for the genetic engineering of Corynebacteria. In Fermentation
Biotechnology, ACS Symposium Series vol. 862, pp. 175-191. Edited by B. C. Saha. Washington, DC: American Chemical Society.

Nothaft, H., Parche, S., Kamionka, A. \& Titgemeyer, F. (2003). In vivo analysis of HPr reveals a fructose-specific phosphotransferase system that confers high-affinity uptake in Streptomyces coelicolor. J Bacteriol 185, 929-937.

Okino, S., Inui, M. \& Yukawa, H. (2005). Production of organic acids by Corynebacterium glutamicum under oxygen deprivation. Appl Microbiol Biotechnol 68, 475-480.

Parche, S., Burkovski, A., Sprenger, G. A., Weil, B., Kramer, R. \& Titgemeyer, F. (2001a). Corynebacterium glutamicum, a dissection of the PTS. J Mol Microbiol Biotechnol 3, 423-428.

Parche, S., Thomae, A. W., Schlicht, M. \& Titgemeyer, F. (2001b). Corynebacterium diphtheriae, a PTS view to the genome. J Mol Microbiol Biotechnol 3, 415-422.

Patek, M., Nesvera, J., Guyonvarch, A., Reyes, O. \& Leblon, G. (2003). Promoters of Corynebacterium glutamicum. J Biotechnol 104, 311-323.

Plumbridge, J. (1999). Expression of the phosphotransferase system both mediates and is mediated by Mlc regulation in Escherichia coli. Mol Microbiol 33, 260-273.

Postma, P. W., Lengeler, J. W. \& Jacobson, G. R. (1993). Phosphoenolpyruvate, carbohydrate phosphotransferase systems of bacteria. Microbiol Rev 57, 543-594.

Stülke, J., Martin-Verstraete, I., Zagorec, M., Rose, M., Klier, A. \& Rapoport, G. (1997). Induction of the Bacillus subtilis ptsGHI operon by glucose is controlled by a novel antiterminator, GlcT. Mol Microbiol 25, 65-78.

Suzuki, N., Okai, N., Nonaka, H., Tsuge, Y., Inui, M. \& Yukawa, H. (2006). High-throughput transposon mutagenesis of Corynebacterium glutamicum and construction of a single-gene disruptant mutant library. Appl Environ Microbiol 72, 3750-3755.

Tanaka, Y., Kimata, K., Inada, T., Tagami, H. \& Aiba, H. (1999). Negative regulation of the pts operon by Mlc: mechanism underlying glucose induction in Escherichia coli. Genes Cells 4, 391-399.

Vadeboncoeur, C., Frenette, M. \& Lortie, L. A. (2000). Regulation of the pts operon in low G+C Gram-positive bacteria. J Mol Microbiol Biotechnol 2, 483-490.

Viana, R., Monedero, V., Dossonnet, V., Vadeboncoeur, C., PerezMartinez, G. \& Deutscher, J. (2000). Enzyme I and HPr from Lactobacillus casei, their role in sugar transport, carbon catabolite repression and inducer exclusion. Mol Microbiol 36, 570-584.

Yokota, A. \& Lindley, N. D. (2005). Central metabolism, sugar uptake and conversion. In Handbook of Corynebacterium glutamicum, pp. 215-240. Edited by L. Eggeling \& M. Bott. Boca Raton, FL: CRC Press.

Yukawa, H., Omumasaba, C. A., Nonaka, H., Kos, P., Okai, N., Suzuki, N., Suda, M., Tsuge, Y., Watanabe, J. \& other authors (2007). Comparative analysis of the Corynebacterium glutamicum group and complete genome sequence of strain R. Microbiology 153, 1042-1058.

Edited by: T. Nihira 